\title{
Comparative genomic analysis of Lactobacillus plantarum GB-LP4 and identification of evolutionarily divergent genes in high-osmolarity environment
}

\author{
Jaehoon Jung ${ }^{1,2} \cdot K_{\text {Kwondo } \mathrm{Kim}^{2,3} \cdot \text { DongAhn Yoo }}^{2,3} \cdot$ Chanho Lee $^{4} \cdot$ Jungsun Kang ${ }^{4} \cdot \mathrm{Ky}^{2}$ ungjin Cho ${ }^{4}$. \\ Dae-Kyung Kang ${ }^{5} \cdot$ Woori Kwak ${ }^{2,3} \cdot$ Sook Hee Yoon ${ }^{1} \cdot$ Hawsun Sohn $^{6} \cdot$ Heebal Kim $^{1,2,3} \cdot$ Seoae Cho ${ }^{2}$
}

Received: 7 May 2016 / Accepted: 27 April 2017 / Published online: 16 November 2017

(c) The Author(s) 2017. This article is an open access publication

\begin{abstract}
Lactobacillus plantarum is one of the widely-used probiotics and there have been a large number of advanced researches on the effectiveness of this species. However, the difference between previously reported plantarum strains, and the source of genomic variation among the strains were not clearly specified. In order to understand further on the molecular basis of L. plantarum on Korean traditional fermentation, we isolated the L. plantarum GB-LP4 from Korean fermented vegetable and conducted whole genome assembly. With comparative genomics approach, we identified the candidate genes that are expected to have undergone evolutionary acceleration. These genes have been reported to associate with the maintaining homeostasis, which are generally known to overcome instability in external environment including low $\mathrm{pH}$ or high osmotic pressure. Here, our results provide an evolutionary relationship between $L$. plantarum species and elucidate the candidate genes that play a pivotal role in evolutionary acceleration of GB-LP4 in high osmolarity environment. This study may provide guidance for further studies on L. plantarum.
\end{abstract}

Keywords Lactobacillus plantarum $\cdot$ Genome $\cdot$ Comparative genomics $\cdot$ Bacterial evolution $\cdot \mathrm{dN} / \mathrm{dS}$

Electronic supplementary material The online version of this article (doi:10.1007/s13258-017-0555-2) contains supplementary material, which is available to authorized users.

Seoae Cho

seoae@cnkgenomics.com

Jaehoon Jung

motto@snu.ac.kr

Heebal Kim

heebal@snu.ac.kr

1 Department of Agricultural Biotechnology and Research Institute of Population Genomics, Seoul National University, Seoul 151-742, Republic of Korea

2 C\&K Genomics, 26 Beobwon-ro 9-gil Bldg. C, \#1008 (H business park) Songpa-gu, Seoul 05836, Republic of Korea

3 Interdisciplinary Program in Bioinformatics, Seoul National University, Seoul 151-742, Republic of Korea

4 Genebiotech Co. Ltd., Seocho-Gu, Seoul 137-787, Republic of Korea

5 Department of Animal Resources Science, Dankook University, Cheonan, Republic of Korea

6 Cetacean Research Institute, National Institute of Fisheries Science, Nam-gu, Ulsan 44780, Republic of Korea

\section{Introduction}

The major group of the lactic acid bacteria, Lactobacillus is commonly found in the human gastrointestinal-tract (De Vries et al. 2006), most of which inhabit in human body are widely known for their effectiveness. A number of previous studies also reported that Lactobacillus is showing anti-viral activities in animals and humans (Kechaou et al. 2013; Maeda et al. 2009; Park et al. 2013). Moreover, the characteristics of Lactobacillus plantarum strains that attract people's attention include their antioxidant activities (Bested et al. 2013).

As one of the most well-known species of lactobacillus, L. plantarum is commonly found in fermented foods. Because many fermented foods are made through pickling process like sprinkling salts, bacteria which inhabit in fermented foods needs to be able to withstand extremely high osmolarity environment. While previous studies revealed that lactic acid bacteria have high survival rate on extreme environment (Chang et al. 2010; Conway et al. 1987; Shah and Jelen 1990), genetic causation of survival related to 
osmolarity or genomic differences between species have not been studied well in plantarum species.

In this study, we sequenced complete genome of $L$. plantarum GB-LP4, isolated from traditional Korean fermented vegetable. The whole genome assembly of the complete genome sequence of $L$. plantarum GB-LP4 was conducted which revealed the genomic contents of $L$. plantarum. Phylogenetic analysis revealed the evolutionary relationship between $L$. plantarum GB-LP4 and other previously reported $L$. plantarum strains. In addition, comparative analysis was performed with other 12 complete genome sequences of L. plantarum strains. From this, we identified evolutionarily accelerated genes that function as transferases. Also, we found the genome of GB-LP4 strains are $97.7 \%$ identical to the $\mathrm{ZJ} 316$ strain isolated from feces of a healthy infant. These results can expand our understanding on the environmental adaptation of $L$. plantarum and provide alternative ways to make use of the L. plantarum GB-LP4 derived from Korean fermented vegetable.

\section{Materials and methods}

\section{Strain isolation and whole genome sequencing}

Extraction of genomic DNA of GB-LP4 strain was proceded and it was purified using UltraClean Microbial DNA Isolation Kit (MoBio, Carlsbad, CA, USA) according to the manufacturer's protocol. The concentration and purity of the isolated DNA was determined using NanoDrop spectrophotometer (Thermo Scientific, Wilmington, DE, USA). Approximately $5 \mu \mathrm{g}$ of the extracted genomic DNA was sheared mechanically into 8-12 kb fragments using Hydroshear system (Digilab, Marlborough, MA, USA). SMRTbell libraries were prepared for SMRT sequencing with C4 chemistry on a PacBio RS $\alpha$ (Pacific Biosciences, Menlo Park, CA, USA). Purification of the libraries was conducted using $0.45 \times$ AMPure XP beads to remove short inserts sized under $1.5 \mathrm{~kb}$. The size distribution of the sheared DNA template was characterized using an Agilent 12000 DNA kit (Applied Biosystems, Santa Clara, CA, USA). The sequencing primers were annealed to the templates at a final concentration of $5 \mathrm{nM}$ template DNA, and DNA polymerase enzyme C4 was added according to the manufacturer's recommendations for small-scale libraries. A DNA/Polymerase Binding Kit P6 (Pacific Biosciences) was used to load the enzyme template-complexes and libraries onto 75,000 zero-mode waveguides (ZMWs). The DNA sequencing reagent 2.0 kit (Pacific Biosciences) was used to sequence SMRT cells using a 120-min sequence capture protocol a long with a stage start to maximize the subread length with PacBio RS $\alpha$.

\section{Genome assembly and annotation}

Raw sequence data was filtered and assembled using SMRT portal system. Due to the high error rate of this pre-assembled data, repetitive remapping and polishing process was carried out by $100 \%$ consensus accuracy. A tool "Circlator" was used to circularize the genome (Hunt et al. 2015). We used RAST annotation system to annotate our genome (Aziz et al. 2008). For functional annotation of annotated genome, COG database (Galperin et al. 2015) was used and annotation map was generated using DNA Plotter (Carver et al. 2009).

\section{Comparative genomics analysis}

A total of 12 complete genome sequences, and $11 \mathrm{draft}$ genome sequences (which is scaffold level) of L. plantarum strains were downloaded from NCBI database (http://www.ncbi.nlm.nih.gov/genome/genomes/1108) for comparative analysis. Average nucleotide identity (ANI) value was calculated for these 24 strains using JSpecies v1.2.1 (Richter and Rosselló-Móra 2009). In order to identify further difference of $L$. plantarum GB-LP4 from other plantarum strains, we built Ortholog gene set for 13 complete genome using MESTORTHO method (Kim et al. 2008). Using PRANK (Löytynoja and Goldman 2008), multiple sequence alignment was run for each ortholog gene. Then, GBlocks (Talavera and Castresana 2007) was used to remove the poorly aligned sites. Finally, a total of 1681 orthologous gene sets were constructed and MEGA7 (Kumar et al. 2016) was used to build phylogenetic tree with neighbor joining method. In addition, bootstrap analysis was performed on the combined data set sequences. The maximum likelihood method (codeml of PAML4) (Yang 2007) was used to estimate the ratio of the rate of non-synonymous substitution to the rate of synonymous substitution, and evolutionarily accelerated genes based on branch and branch-site model. Visualization of multiple sequence alignment in evolutionarily accelerated gene from branch-site model was conducted using WebLogo3 (Crooks et al. 2004). Further comparative analysis with ZJ316 was conducted using Artemis comparison tool (Carver et al. 2005).

\section{Complete genome sequence accession number}

The complete genome sequence of $L$. plantarum GB-LP4 have been deposited with the NCBI database under the accession number SUB1431878. 


\section{Results}

\section{General features of $L$. plantarum GB-LP4 genome}

The genome of $L$. plantarum GB-LP4 was composed of a single circular DNA chromosome of 3,204,876 b.p. with 44.7\% GC contents (Table 1). This genome contained 3,204 CDS (ORFs) and 85 RNAs (69 tRNA and 10 rRNA, 65S rRNA). Among the predicted ORFs, 756 genes (23.5\%) were unknown or hypothetical genes (Fig. 1). The remainders of 2448 genes $(76.5 \%)$ were expected to work as functional genes. Categorization of ORFs based on the SEED subsystem categorization and $\mathrm{COG}$ functional categorization are shown in Fig. 2.

Among 2448 genes, 1908 ORFs were categorized into 25 SEED subsystem categories. The subsystem "cofactors, vitamins, prosthetic groups, pigments" was assigned with 129 ORFs and 10 ORFs in this subsystem were related to Thiamin biosynthesis, which increases as the Korean traditional fermented vegetable ripens (Lee 1991). In addition, there were 49 ORFs, which were categorized into virulence, disease and defense. Among these ORFs, function of resistance to antibiotics and toxic compounds are most abundant (35 of 49). According to COG functional database, 2610 ORFs ( $81.4 \%$ of totally predicted ORFs) were assigned to COG functional categories. Among these, $1052 \mathrm{ORFs}$ were included in the major five COG functional categories: 208 ORFs in category E (amino acid transport and metabolism), 255 ORFs in category $\mathrm{G}$ (carbohydrate transport and metabolism), 171 ORFs in category M (cell wall/membrane/envelope biogenesis), 298 ORFs in category K (transcription), and 120 ORFs in category $\mathrm{J}$ (translation, ribosomal structure and biogenesis).

\section{Comparative phylogenetic tree analysis}

For comparative tree analysis of GB-LP4 strain, two ANI trees and one phylogenetic tree were built. The two trees based on ANI values were constructed with 24 available genome sequences and 13 available complete genome

Table 1 General features of L. plantarum strains

\begin{tabular}{lllll}
\hline Strain & GB-LP4 & ZJ316 & P8 & 16 \\
\hline Genome size (b.p.) & $3,204,876$ & $3,203,964$ & $3,035,719$ & $3,044,678$ \\
GC contents (\%) & 44.7 & 44.6 & 44.5 & 44.7 \\
Open reading frames & 3204 & 3103 & 3080 & 3148 \\
Functional genes & 2448 & 2317 & 2216 & 2243 \\
tRNA & 69 & 61 & 68 & 66 \\
rRNA & 16 & 15 & 16 & 16 \\
ANI (\%) & 100 & 99.94 & 99.15 & 99.23 \\
\hline
\end{tabular}

sequences in NCBI database, respectively (Fig. 3). In every graph, four strains-P8, 16, UCMA3037, ZJ316-were grouped with GB-LP4 while ZJ316 being the closest strain to the GB-LP4 (99.94\% of ANI value). The general features of these four strains are shown in the Table 1 for a comparison. ZJ316, which was isolated from feces of a healthy infant, was shown to have effects on pig growth and pork quality in previous study and it is predicted to inhibit of the growth of pathogens and promote increase in villus height. The phylogenetic tree was constructed using 1681 orthologous gene sets for 13 complete genome sequences. The tree was generated using neighbor joining method with bootstrap 1000 times (Fig. 3). This phylogenetic tree's overall pattern was slightly different from ANI tree, and still the closest strain of GB-LP4 was ZJ316.

\section{Comparative genomics analysis}

To reveal the evolutionary relationship between strains, we conducted $\mathrm{dN} / \mathrm{dS}$ analysis using two models (branch, branch-site) and the results are shown in Table 2. In $\mathrm{dN} / \mathrm{dS}$ analysis based on branch model, 2 genes [acetyltransferase (isoleucine patch superfamily), 2-C-methyl-D-erythritol 4-phosphate cytidylyltransferase] were identified as evolutionarily accelerated genes. In $\mathrm{dN} / \mathrm{dS}$ analysis based on branch site model, only one gene was identified as evolutionarily accelerated gene. This gene functions as aspartate aminotransferase.

For further comparative genomic analysis, comparative genomic analysis of GB-LP4 and its closest strain ZJ316 was conducted. The result showed that nearly $97.7 \%$ $(3,132,698 / 3,204,876$ b.p.) sequences in GB-LP4 had $100 \%$ identity to ZJ316 and matching regions were shown in Fig. S1. We focused on the region that had relatively low identity $(1,145,354-1,150,000$ b.p., lower than $80 \%$ identity) and found that this region is known for the function of cardiolipin synthase in GB-LP4. Although the gene encoding cardiolipin synthase was found in other regions in the two strains, there are two genes encoding cardiolipin synthase in GB-LP4 whereas ZJ316 has only one gene encoding cardiolipin synthase.

\section{Discussion}

The method of Korean-traditional fermentation involves the pickling process, which attenuates the number of microbes by hyperosmolarity. Nonetheless, GB-LP4 could survive in this high-osmolarity environment. Interestingly, our findings are closely associated with the GB-LP4's adaptation to the high-stress environment. In branch model, a gene encoding 2-C-methyl-D-erythritol 4-phosphate cytidylyltransferase (EC 2.7.7.60) was identified to be positively selected. 


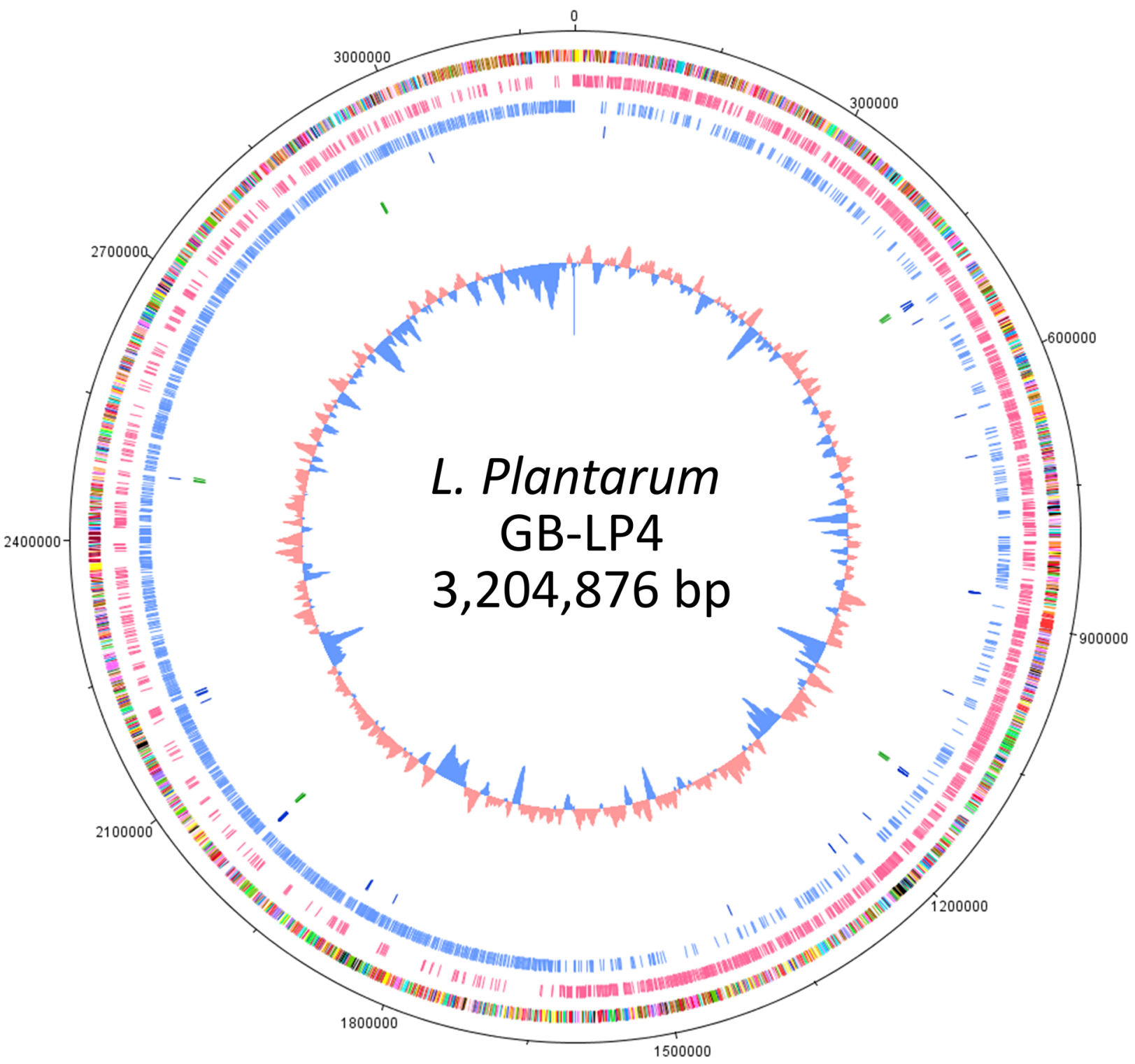

$\begin{array}{ll}\text { O } & \text { Posttranslational modification, protein turnover, chaperones } \\ \text { P } & \text { Inorganic ion transport and metabolism } \\ \text { Q } & \text { Secondary metabolites biosynthesis, transport and catabolism } \\ \text { R } & \text { General function prediction only } \\ \text { S } & \text { Function unknown } \\ \text { T } & \text { Signal transduction mechanisms } \\ \text { U } & \text { Intracellular trafficking, secretion, and vesicular transport } \\ \text { V } & \text { Defense mechanisms } \\ \text { W } & \text { Extracellular structures } \\ \mathrm{X} & \text { Mobilome: prophages, transposons } \\ \text { Mu } & \text { Multiple Class }\end{array}$

Fig. 1 Genome map of L. plantarum GB-LP4. From outer circle to inner circle, each indicates the COG distributions, CDS in leading strand, CDS in lagging strand, tRNA, rRNA and GC contents ratio. Functional genes are labeled around the outer circle as follow 
(a)
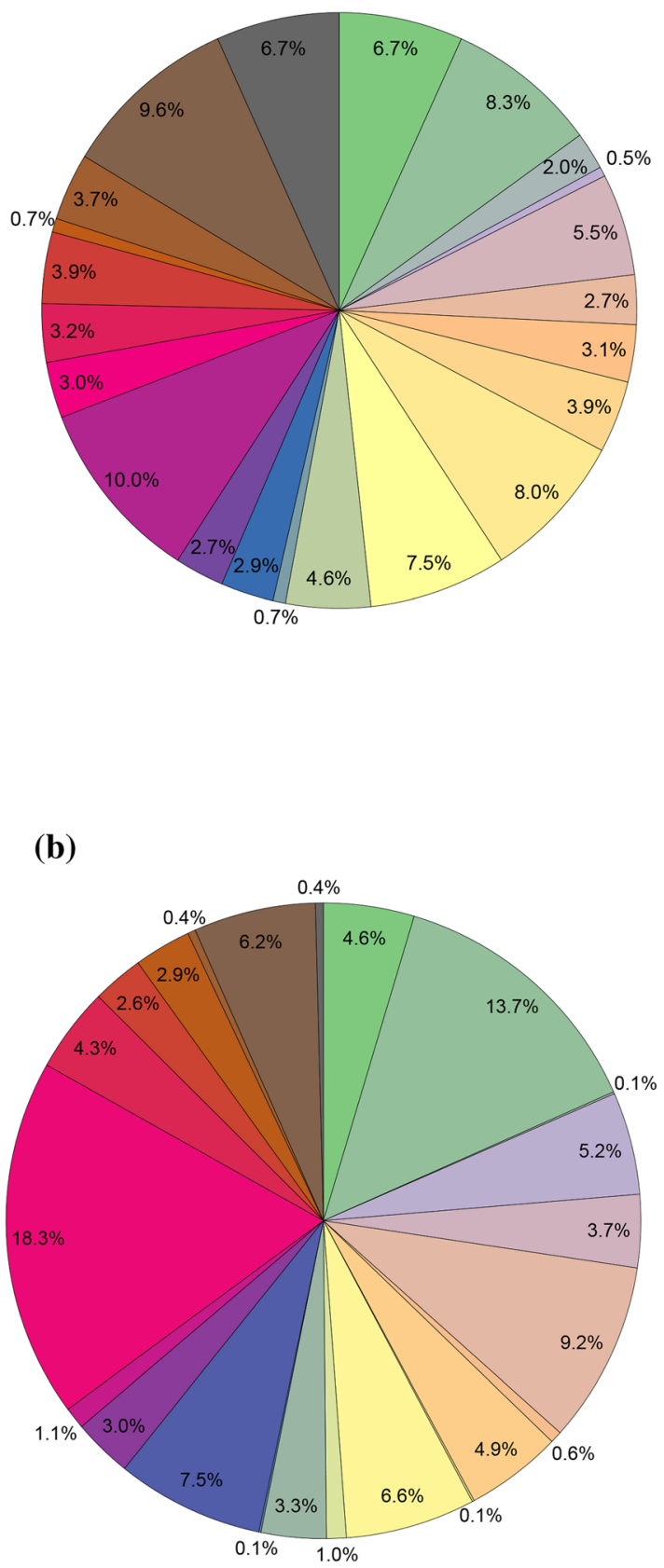

Amino acid transport and metabolism

Carbohydrate transport and metabolism

Cell cycle control, cell division, chromosome partitioning

Cell motility

Cell wall/membrane/envelope biogenesis

Coenzyme transport and metabolism

Defense mechanisms

Energy production and conversion

Function unknown

General function prediction only

Inorganic ion transport and metabolism

Intracellular trafficking, secretion, and vesicular transport

Lipid transport and metabolism

Mobilome: prophages, transposons

Mulitple class

Nucleotide transport and metabolism

Posttranslational modification, protein turnover, chaperones

Replication, recombination and repair

Secondary metabolites biosynthesis, transport and catabolism

Signal transduction mechanisms

Transcription

Translation, ribosomal structure and biogenesis

Amino Acids and Derivatives

Carbohydrates

Category

Cell Division and Cell Cycle

Cofactors, Vitamins, Prosthetic Groups, Pigments

DNA Metabolism

Dormancy and Sporulation

Fatty Acids, Lipids, and Isoprenoids

Iron acquisition and metabolism

Membrane Transport

Metabolism of Aromatic Compounds

Miscellaneous

Motility and Chemotaxis

Nucleosides and Nucleotides

Phages, Prophages, Transposable elements, Plasmids

Potassium metabolism

Protein Metabolism

Regulation and Cell signaling

Respiration

RNA Metabolism

Secondary Metabolism

Stress Response

Virulence, Disease and Defense

Fig. 2 Functional categorization of all predicted ORFs in the genome of the strain GB-LP4 based on a COG and $\mathbf{b}$ SEED databases. The pie charts present that the proportion of functional categorization of ORFs in the genome based on two different databases

This enzyme catalyzes the formation of 4-diphosphocytidyl-2-C-methyl-D-erythritol from cytidine triphosphate and 2-C-methyl-D-erythritol 4-phosphate, and is involved in the non-mevalonate pathway of isoprenoid biosynthesis leading to the formation of isopentenyl pyrophosphate
(IPP) and dimethylallyl pyrophosphate (DMAPP) (Kuzuyama and Seto 2012). In bacteria, Isoprenoid functions as cell wall biosynthesis intermediates. The gene most likely to have undergone positive selection in branch-site model was aspartate aminotransferase (EC 2.6.1.1). According to 


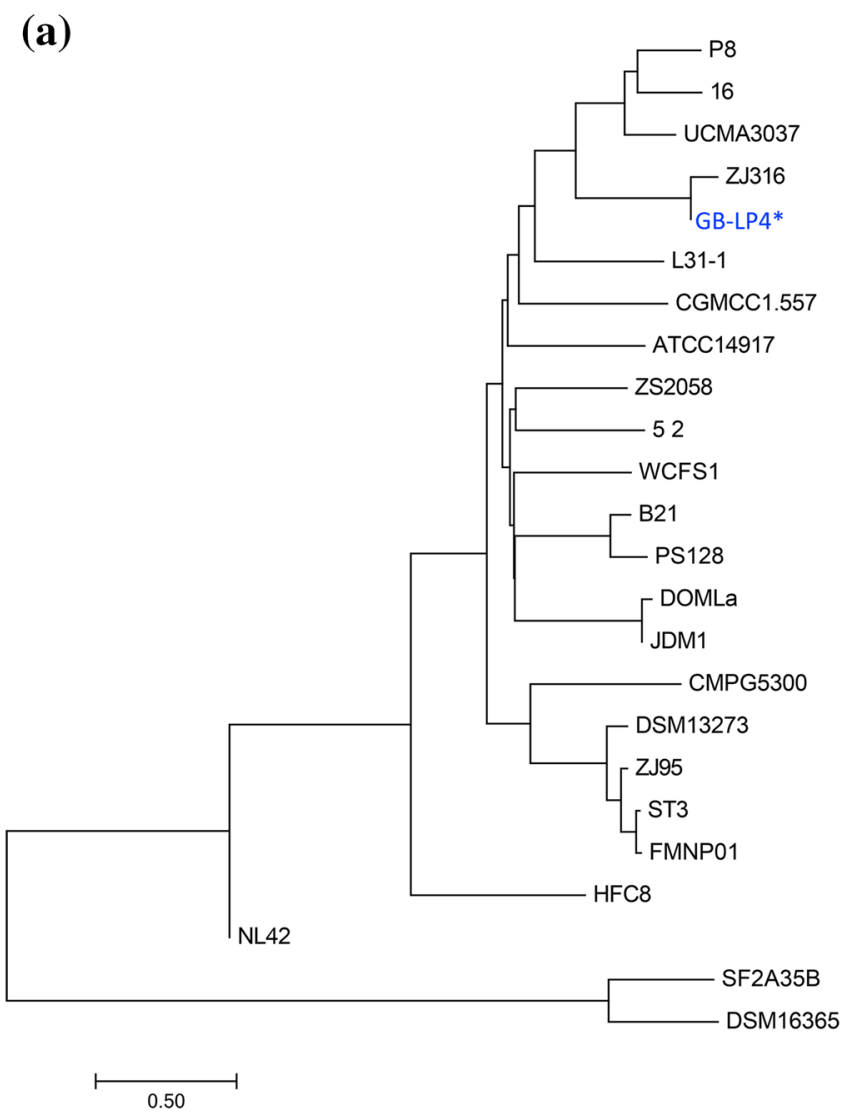

Fig. 3 Comparative tree analysis. a ANI tree analysis of 24 genome sequences in $L$. plantarum strain using JSpecies. b ANI tree analysis of GB-LP4 with 12 available complete genome sequences of L. plan- (b)

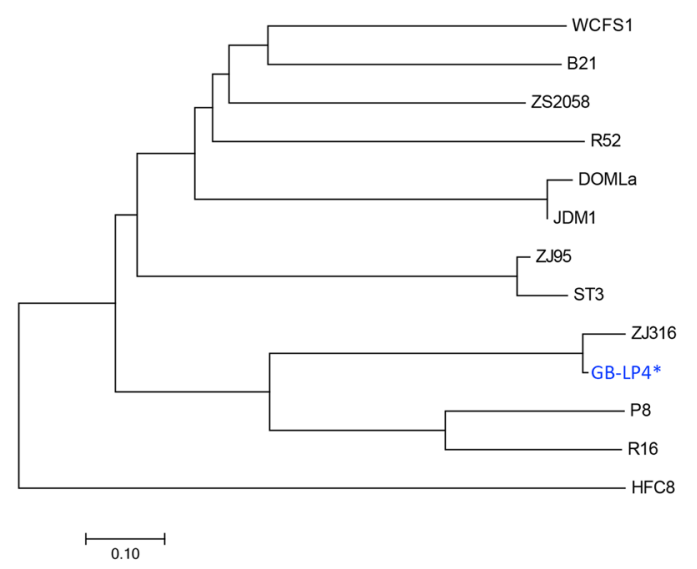

(c)

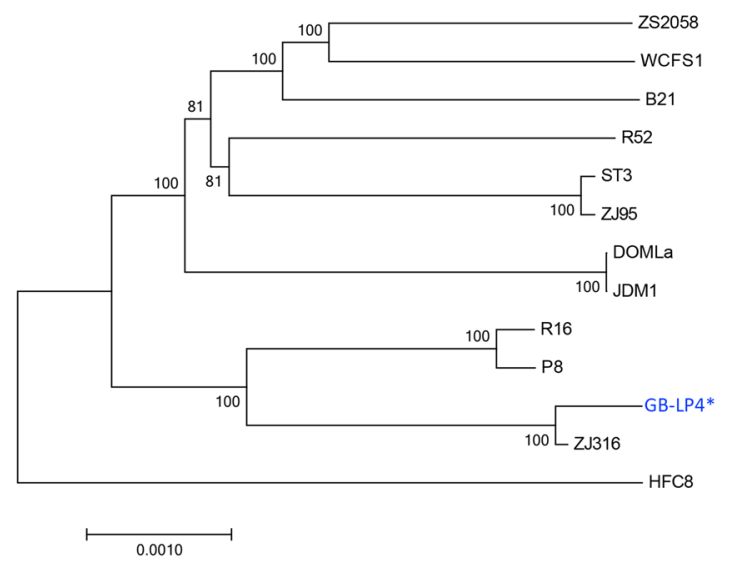

tarum. c Phylogenetic tree analysis of GB-LP4 with seven available complete genome sequences using ortholog gene sets

Table 2 Significant genes and relevant information in dN/dS analysis of branch model and branch-site model

\begin{tabular}{llll}
\hline Branch model & & & \\
\hline Function & $\omega_{\text {F.G. }}$ & $\omega_{\text {B.G. }}$ & FDR \\
\hline Acetyltransferase (isoleucine patch superfamily) & 425.9473 & 0.0266 & $2.77 \mathrm{E}-06$ \\
$\begin{array}{llll}\text { 2-C-methyl-D-erythritol 4-phosphate cytidylyl- } \\
\text { transferase }\end{array}$ & 999 & 0.0363 & $2.06 \mathrm{E}-03$ \\
\hline Branch-site model & & & $\omega 2 \mathrm{a}_{\text {B.G. }}$ \\
\hline Function & Proportion of 2a & $\omega 2_{\text {F.G. }}$ & FDR \\
\hline Aspartate aminotransferase (EC 2.6.1.1) & 0.0918 & 6.9596 & $2.91 \mathrm{E}-14$ \\
\hline
\end{tabular}

previous studies, decarboxylation of aspartate to alanine was found to take part in the generation of metabolic energy and regulation of intracellular $\mathrm{pH}$ in Lactobacillus (Abe et al. 1996; Konings et al. 1995). Concerning these observed properties, we speculated that aspartate aminotransferase might have a role related to maintaining homeostasis in high-osmolarity environment. Moreover, we have found that the gene encoding for cardiolipin synthase is located in the relatively low identity site between ZJ316 and GB-LP4. Osmotic induction of the gene encoding cardiolipin synthase contributes to the increasing proportion of cardiolipin as the proportion of phosphatidylethanolamine decreases in bacteria (Romantsov et al. 2009). Also, previous study found that the absence of cardiolipin synthase lead to significant uncoupling at the maximal rate of respiration and osmotic instability because of the strong interaction of cardiolipin 
with respiratory complexes (Koshkin and Greenberg 2002). From these findings, we speculated that the altered structure of evolutionarily selected genes in GB-LP4 would help to overcome instability in high osmolarity environment.

In conclusion, we performed comparative analysis on GB-LP4 and identified evolutionarily accelerated genes that are related to the survival of GB-LP4 in the specific environment-Korean traditional fermented vegetable. Further research on these genes will contribute to the understanding of adaptation of bacterial strains to a specific environment and the resulting phenotypic changes in bacteria.

Acknowledgements This work was supported by a grant from the Next-Generation BioGreen 21 Program (PJ01115901), Rural Development Administration, Republic of Korea and the National Institute of Fisheries Science (R20160029).

\section{Compliance with ethical standards}

Conflict of interest Jaehoon Jung, Kwondo Kim, DongAhn Yoo, Chanho Lee, Jungsun Kang, Kyungjin Cho, Dae-Kyung Kang, Woori Kwak, Sook Hee Yoon, Hawsun Sohn, Heebal Kim, and Seoae Cho declare no conflict of interest on the contents of the manuscript.

Research involving human and animal rights This article does not contain any studies with human subjects or animals performed by any of the authors.

Open Access This article is distributed under the terms of the Creative Commons Attribution 4.0 International License (http://creativecommons.org/licenses/by/4.0/), which permits unrestricted use, distribution, and reproduction in any medium, provided you give appropriate credit to the original author(s) and the source, provide a link to the Creative Commons license, and indicate if changes were made.

\section{References}

Abe K, Hayashi H, Maloney PC (1996) Exchange of aspartate and alanine mechanism for development of a proton-motive force in bacteria. J Biol Chem 271:3079-3084

Aziz RK, Bartels D, Best AA, DeJongh M, Disz T, Edwards RA, Formsma K, Gerdes S, Glass EM, Kubal M (2008) The RAST Server: rapid annotations using subsystems technology. BMC Genomics 9:75

Bested AC, Logan AC, Selhub EM (2013) Intestinal microbiota, probiotics and mental health: from Metchnikoff to modern advances: part II-contemporary contextual research. Gut Pathog 5:1

Carver TJ, Rutherford KM, Berriman M, Rajandream M-A, Barrell BG, Parkhill J (2005) ACT: the Artemis comparison tool. Bioinformatics 21:3422-3423

Carver T, Thomson N, Bleasby A, Berriman M, Parkhill J (2009) DNAPlotter: circular and linear interactive genome visualization. Bioinformatics 25:119-120

Chang JH, Shim Y, Cha SK, Chee K (2010) Probiotic characteristics of lactic acid bacteria isolated from kimchi. J Appl Microbiol 109:220-230
Conway P, Gorbach S, Goldin B (1987) Survival of lactic acid bacteria in the human stomach and adhesion to intestinal cells. J Dairy Sci 70:1-12

Crooks GE, Hon G, Chandonia J-M, Brenner SE (2004) WebLogo: a sequence logo generator. Genome Res 14:1188-1190

De Vries MC, Vaughan EE, Kleerebezem M, de Vos WM (2006) Lactobacillus plantarum - survival, functional and potential probiotic properties in the human intestinal tract. Int Dairy J 16:1018-1028

Galperin MY, Makarova KS, Wolf YI, Koonin EV (2015) Expanded microbial genome coverage and improved protein family annotation in the COG database. Nucleic Acids Res 43:D261-D269

Hunt M, De Silva N, Otto TD, Parkhill J, Keane JA, Harris SR (2015) Circlator: automated circularization of genome assemblies using long sequencing reads. Genome Biol 16:1

Kechaou N, Chain F, Gratadoux J-J, Blugeon S, Bertho N, Chevalier C, Le Goffic R, Courau S, Molimard P, Chatel JM (2013) Identification of one novel candidate probiotic Lactobacillus plantarum strain active against influenza virus infection in mice by a largescale screening. Appl Environ Microbiol 79:1491-1499

Kim KM, Sung S, Caetano-Anollés G, Han JY, Kim H (2008) An approach of orthology detection from homologous sequences under minimum evolution. Nucleic Acids Res 36:e110-e110

Konings WN, Lolkema JS, Poolman B (1995) The generation of metabolic energy by solute transport. Arch Microbiol 164:235-242

Koshkin V, Greenberg ML (2002) Cardiolipin prevents rate-dependent uncoupling and provides osmotic stability in yeast mitochondria. Biochem J 364:317-322

Kumar S, Stecher G, Tamura K (2016) MEGA7: molecular evolutionary genetics analysis version 7.0 for bigger datasets. Mol Biol Evol 33:1870-1874

Kuzuyama T, Seto H (2012) Two distinct pathways for essential metabolic precursors for isoprenoid biosynthesis. Proc Jpn Acad B $88: 41-52$

Lee YC (1991) Kimchi: the famous fermented vegetable product in Korea. Food Rev Int 7:399-415

Löytynoja A, Goldman N (2008) Phylogeny-aware gap placement prevents errors in sequence alignment and evolutionary analysis. Science 320:1632-1635

Maeda N, Nakamura R, Hirose Y, Murosaki S, Yamamoto Y, Kase T, Yoshikai Y (2009) Oral administration of heat-killed Lactobacillus plantarum $\mathrm{L}-137$ enhances protection against influenza virus infection by stimulation of type I interferon production in mice. Int Immunopharmacol 9:1122-1125

Park M-K, Vu N, Kwon Y-M, Lee Y-T, Yoo S, Cho Y-H, Hong S-M, Hwang HS, Ko E-J, Jung Y-J (2013) Lactobacillus plantarum DK119 as a probiotic confers protection against influenza virus by modulating innate immunity. PLoS ONE 8:e75368

Richter M, Rosselló-Móra R (2009) Shifting the genomic gold standard for the prokaryotic species definition. Proc Natl Acad Sci USA 106:19126-19131

Romantsov T, Guan Z, Wood JM (2009) Cardiolipin and the osmotic stress responses of bacteria. Biochim Biophys Acta 1788:2092-2100

Shah N, Jelen P (1990) Survival of lactic acid bacteria and their lactases under acidic conditions. J Food Sci 55:506-509

Talavera G, Castresana J (2007) Improvement of phylogenies after removing divergent and ambiguously aligned blocks from protein sequence alignments. Syst Biol 56:564-577

Yang Z (2007) PAML 4: phylogenetic analysis by maximum likelihood. Mol Biol Evol 24:1586-1591 